Rapport - Société canadienne d'histoire de l'Église catholique

\title{
Le développement de l'Église Catholique à Sudbury
}

\section{Lorenzo Deschamps}

Volume 27, 1960

URI : https://id.erudit.org/iderudit/1007401ar

DOI : https://doi.org/10.7202/1007401ar

Aller au sommaire du numéro

Éditeur(s)

La Société canadienne d'histoire de l'Église catholique

ISSN

0318-6148 (imprimé)

1927-7075 (numérique)

Découvrir la revue

Citer cet article

Deschamps, L. (1960). Le développement de l'Église Catholique à Sudbury. Rapport - Société canadienne d'histoire de l'Église catholique, 27, 23-33.

https://doi.org/10.7202/1007401ar

Tous droits réservés @ La Société canadienne d'histoire de l'Église catholique, 1961
Ce document est protégé par la loi sur le droit d'auteur. L'utilisation des services d'Érudit (y compris la reproduction) est assujettie à sa politique d'utilisation que vous pouvez consulter en ligne.

https://apropos.erudit.org/fr/usagers/politique-dutilisation/ 


\section{Le développement de l'Église Catholique à Sudbury}

A l'occasion de ce Congrès, le Révérend Père Cadieux, directeur de la Société Historique, m'a confié la délicate tâche de traiter du développement du catholicisme à Sudbury.

La consultation des archives du Collège du Sacré-Cœur, les renseignements puisés dans le Canada Ecclésiastique et auprès des bienveillants curés des différentes paroisses ainsi que l'aide soutenue du Père Cadieux, me permettent de faire revivre une page d'histoire locale.

Les précieuses découvertes effectuées par mes recherches font réaliser la vérité de ce vers du poète: " 0 notre histoire, écrin de perles ignorées!»

En effet, lorsqu'en considérant la circonscription du diocèse du Sault-Sainte-Marie, dont Sudbury est fier de faire partie, nous réalisons l'immense étendue des districts de Sudbury et d'Algoma; la portion du district de Nipissing au nord et à l'ouest du lac Nipissing et les Iles Manitoulin; qui de nous s'arrêtent pour penser à son origine? Rares sont-ils? Si notre diocèse, érigé le 16 septembre 1904 par son Excellence $\mathrm{M}^{\mathrm{gr}}$ D. J. Scollard (1905-1934), remplacé par son Excellence $\mathbf{M}^{\mathrm{gr}}$ Ralph Hubert Dignan et son Excellence $\mathrm{M}^{\mathrm{gr}}$ Alexander Carter, coadjuteur, compte 152,041 catholiques, 116 prêtres séculiers, 66 prêtres réguliers, 73 églises avec prêtres résidant, 6 collèges, 2 académies, 7 hôpitaux, 190 écoles catholiques primaires, 9 écoles secondaires ainsi que 2 écoles industrielles pour les Indiens, à qui le devons-nous? Qui nous a légué un si bel héritage? Les archives du Collège nous répondent: "Le 16 septembre 1904, le diocèse du Sault-Sainte-Marie était fondé, englobant les districts d'Algoma, de la Baie du Tonnerre, la portion au nord et à l'ouest de celui de Nipissing et les Iles Manitoulin. La principale partie chevauchait le long de la voie ferrée du Pacifique canadien de North Bay à Fort William. Dans cet immense rectangle habitaient 31,064 catholiques: 20,064 Canadiens français, 6,000 de différentes nationalités et 5,000 Indiens. Il comptait 64 églises. Les Jésuites, qui en avaient bâti 58, dirigeaient 14 paroisses sur 19 ainsi que toutes les missions indiennes. $\gg$

Depuis soixante ans, ces fils de saint Ignace évangélisaient la région. Ils avaient à peu près exclusivement fondé les missions et les paroisses qu'ils cédaient l'une après l'autre à leur évêque, participant ainsi largement à l'érection et à l'organisation du diocèse. L'évêque de Peterborough, $\mathrm{M}^{\mathrm{gr}}$ Richard 0'Connor en témoignait lui-même en écrivant: "Ce diocèse du Sault Sainte-Marie est grandement redevable aux Pères Jésuites qui ont travaillé avec zèle au salut des âmes. » 
Jusqu'à l'époque de la Confédération, lit-on, les cadres de l'apostolat étaient restreints aux villages indiens en bordure des lacs Huron, Supérieur et Michigan, mais ils s'élargirent lors de la pénétration des Blancs dans le Nouvel Ontario jusqu'à se confondre en 1904 avec les frontières d'un nouveau diocèse, celui du Sault Sainte-Marie. Ce rapide développement religieux invite à rechercher quelles influences ont accéléré le mouvement démographique dans l'Ontario nord.

Un des facteurs de ce développement semble bien être l'industrie forestière qui naissait dans le district d'Algoma vers 1870. Dans les chantiers expioites par les compagnies de bois, le long des rivières dont les eaux baignent l'Ile Manitoulin et se jettent dans le Canal du Nord, afflua un grand nombre de Canadiens français qui s'établirent peu à peu sur les terres. Grâce à ces défricheurs audacieux, qui puisaient leur force d'âme près du missionnaire, un nouveau royaume s'ouvrait à l'expansion catholique et française.

Une douzaine d'années plus tard, la découverte d'un immense gisement de nickel attire dans la région de Sudbury des équipes de prospecteurs qui repèrent les mines de Copper-Cliff, Stobie et Creighton. Des acheteurs accourent de partout.

Cependant la construction du chemin de fer Pacifique Canadien devient la principale cause du développement de tout l'Ontario nord. Lorsqu'en 1882, les cheminots de ce chemin de fer pénétrèrent dans le Nouvel Ontario, cette région formait une partie du diocèse de Peterborough. L'évêque, son Excellence $\mathbf{M}^{\mathrm{gr}}$ Joseph Dowling, appelle les Jésuites à son aide. Ceux-ci choisirent Sudbury comme centre de ralliement. Pendant quatre ans, notent les Archives du Collège SacréCour, soit de 1882 à 1886, les pères François-Xavier Santerre, Louis Côté, Hormidas Caron, Richard Baxter, Olivier Neault, Jean-Baptiste Nolin et Joseph Specht furent missionnaires, chapelains des cheminots, visitant tous les postes échelonnés le long de la voie ferrée. Ces missionnaires, chapelle portative au dos ou au bout du bras, voyageant plus souvent à pieds qu'en voiture, laissant des lambeaux de soutanes aux débris des roches dynamitées, étaient bien, pour les Peaux-Rouges comme pour les Blancs, les Robes-Noires, qui ne s'inquiétaient pas des effarouchements de quelques fanatiques ombrageux.

Ils logeaient chez des familles hospitalières mais le plus souvent dans des chantiers malpropres où les attendaient des lits superposés le long des murs. C'est dire qu'ils couchaient sur la dure, roulés dans une couverture sur deux planches garnies de foin. Le vent chantait et giflait le malheureux par les fentes des planches disjointes des murs tandis qu'au travers le toit, les nuages laissaient tomber quelques gouttes de leurs eaux bienfaisantes.

Malgré cette vie rude, éreintante, hérissée de sacrifices, ces courageux missionnaires savaient plaisanter: "J'ai, dit le père Caron, une paroisse large de quatre pieds, et longue de 500 milles. Il me faut, en petite vitesse, deux jours et deux nuits pour la parcourir, dans toute sa longueur, mais je la traverse en deux secondes. " «La mienne, reprend le père Santerre, n'est pas plus large et n'a que 300 milles de long, ce 
qui fait que je n'ai pas d'assistant. Je suis aussi mieux partagé. Vos chars ne sont que des plates-formes qui ne vous protègent pas contre la pluie, le vent ou la neige, moi j'ai des pullmans à boeufs. " Ces innocentes plaisanteries nous en disent plus que de longues pages.

Le commerce du bois surgissant là où les équipes de bûcherons, de cheminots et de constructeurs avaient déblayé les voies, attire bientôt nombre de colons, cheminots ou employés de scieries. Le prêtre érige une chapelle, autour de laquelle comme auprès d'une mère se groupent les cabanes d'ouvriers et de défricheurs. On invite les amis d'en-bas. Voilà que les familles augmentent provenant de l'extérieur mais plus encore de la simple vertu de l'admirable fécondité canadienne. Des agglomérations se forment, des hameaux apparaissent, des villages s'organisent, les paroisses sont nées.

\section{Paroisse Sainte-AnNe.}

Le fait de voir Sudbury choisie par les pères Jésuites comme centre de ralliement, nous devinons facilement que cette ville était destinée à devenir le berceau de la première paroisse de l'Ontario nord: celle qui deviendra la paroisse mère d'où se détacheront les paroisses: Christ The King, Saint-Jean-de-Brébeuf, Saint-Clément, Sainte-Brigitte, Sainte-Trinité, Notre-Dame-du-Bon-Secours, La Toussaint, Saint-Rédempteur, Saint-Eugène, L'Annonciation, Saint-Antoine, Saint-Patrice, Saint-Casimir, Sainte-Marie, St. Andrew et finalement Saint-Dominique.

Nous penchant sur ce berceau, nous la majestueuse forêt de pins qui couvrait, en 1883, les rochers actuellement dénudés de notre florissante ville de Sudbury, voilà pourquoi le nom poétique de Sainte-Annedes-Pins fut primitivement donné au groupement de colons pionniers du Nord. Si la Compagnie du Pacifique canadien lui imposait plus tard, par l'entremise de son premier surintendant des travaux, M. James Worthington, le nom de Sudbury, appellation d'un bourg d'Angleterre, la première église et sa résidence portent encore le nom de la glorieuse patronne des Canadiens français sainte Anne, mentionne les Archives.

C'est le R.P. Joseph Specht, premier prêtre ordonné dans le diocèse le 22 mai 1882 à Fort William, devenu le premier missionnaire de la région, qui, plaçant sa propre paroisse sous le vocable de sainte Anne, la baptisa chrétiennement. C'est lui encore qui célébra la première messe, le 30 mars 1883, dans la cabane construite pour l'éclaireur Jessie Smith.

Cependant le R.P. Jean-Baptiste Nolin en devint le curé fondateur. Ce dernier, comprenant que le zèle apostolique devait non seulement consister à accompagner les équipes d'ouvriers en route vers l'ouest, mais aussi pourvoir aux besoins spirituels des stationnaires qui fixaient leurs demeures en grappes le long de la voie, songea à bâtir une église, ou plutôt une maison qui servirait à la fois de presbytère et de chapelle provisoire. Inutile de dire que les pins ont servi à élever les murs de cette maison qui devait servir jusqu'à la construction de la nouvelle église ouverte en 1889. 
Notons que la première grand-messe fut chantée en plein air le jour de la solennité de l'Assomption de la Sainte Vierge, le 19 août 1883; que le premier mariage fut béni le 12 septembre de la même année et que le premier baptême date du 2 octobre.

A l'automne de 1883 le chantier-chapelle étant trop petit, le père Nolin se mit en frais de construire un presbytère, celui-là même qui sert encore de bureaux aux Pères. On n'y a ajouté qu'une allonge de dix pieds, une fournaise à eau chaude, un lambris de briques et un vestibule viiré.

En 1884, Sudbury comptait déjà une cinquantaine de familles lorsqu'en juin le père Nolin, rappelé à Montréal, est remplacé par le père Louis Côté qui connut des heures pénibles à exercer son ministère parmi des ouvriers Canadiens français et des Européens de toutes nations et de toutes croyances religieuses, ces derniers plus ou moins bien disposés envers le prêtre.

Au printemps de 1884, l'établissement d'une école s'imposait et le Père se mit à l'œuvre. Grâce à une séance dramatique et musicale qui rapporta une soixantaine de dollars, les classes commençaient le 2 avril. La chapelle servait d'école, le sanctuaire ayant été séparé de la nef par une porte à deux battants se repliant sur eux-mêmes. Une pieuse personne, Mlle Smith, assumait la tâche d'institutrice pendant que le Père missionnaire allait presque chaque mois, de porte en porte, amasser ce qui était nécessaire au soutien de l'école.

Sudbury, qui avait pris les allures d'une mission constituée et organisée, avec une population catholique de 376 âmes en 1887, eut l'insigne honneur de recevoir la première visite pastorale de $\mathrm{M}^{\mathrm{gr}}$ Thomas Joseph Dowling de Peterborough. Pour la circonstance, les catholiques avaient pavoisé les rues et élevé des arcs de triomphe, et se portèrent à la rencontre de l'évêque. Le 9 septembre, l'évêque conféra la confirmation à 36 confirmants puis bénit la première pierre de la nouvelle église en construction depuis quatre mois, dans laquelle le père Hormidas Caron pût célébrer la première messe le 5 mai 1889. Avec sa grande église et sa communauté de missionnaires, Sudbury s'élevait au rang de paroisse et l'esprit chrétien allait s'en ressentir. Au père Toussaint Lussier était réservé de voir, durant un supériorat long de douze ans, le plein épanouissement des œuvres civiles et religieuses. L'épreuve ne manqua cependant pas de le visiter, car le Vendredi Saint, 23 mars 1894, son église devenait la proie des flammes. Elle fut reconstruite en quelques mois. Il fallut aussi songer à une nouvelle école. Bâtie durant l'été, elle était prête à recevoir 250 élèves dès la rentrée des classes. Notons que cette école transformée devenait en 1929, l'orphelinat d'Youville.

En 1896, après des pourparlers et des négociations, le père Lussier obtenait trois religieuses des Sœurs Grises de la Croix d'Ottawa pour ouvrir un hôpital qui remplacerait les deux alors existants. En septembre, des religieuses de cette même communauté prenaient charge des classes. 
Depuis 1905, nous pouvons dire que Sudbury, en tant que ville et paroisse, est devenue pleine d'activité et de mouvement. Nous nous contenterons de mentionner l'essor donné aux différentes organisations. En 1905 Sainte-Anne possède l'Apostolat de la Prière. En 1910, le père Lefebvre fonde l'Association Canadienne-française d'Education d'Ontario.

En 1912, le 25 mars, le Général des Jésuites, à Rome, consentit à la fondation d'un collège classique à Sudbury. La construction en fut terminée en avril 1913.

En 1913, le père Paré devient premier directeur de la Ligue du Sacré-Cœur.

Le 17 octobre de la même année s'organise l'Association catholique de la Jeunesse canadienne.

Le 20 octobre 1913, se fonde la Caisse Populaire Sainte-Anne.

En 1917, nous notons la fondation du bulletin paroissial de Sudbury, louable initiative du père Joseph Desjardins, vicaire à SainteAnne. Pendant nombre d'années il couvrira toute la région du Nouvel Ontario jusqu'au Sault Sainte-Marie et rendra d'immenses services au point de vue religieux et français.

Juin 1917 verra la consécration des familles au Sacré-Cœur et la formation des Ligues des Cadets du Sacré-Cœur et des Cadettes de Marie.

Tout comme en 1916, la Saint-Jean-Baptiste sera fêtée d'une façon grandiose et la Société Saint-Jean-Baptiste subsiste encore.

Le 30 mai 1929, $\mathrm{M}^{\mathrm{gr}}$ Louis Rhéaume, évêque de Haileybury, bénissait l'orphelinat.

En mai 1942, le père Rosaire Legault obtenait la fondation de la Fédération des Ligues du Sacré-Cœur ainsi que celle de la confrérie du Tiers-Ordre et devenait lui-même l'un des fondateurs de la Société Historique du Nouvel Ontario.

En 1945, le père Alphonse Deguire organisa des cours de préparation au mariage, patrona les cours de psychologie familiale et des Cercles d'études coopératives.

En 1949 se fondait le Cercle Lacordaire sous la direction du père Alphonse Raymond. Les pères Amédée Dupas et Thomas Migneault prenaient l'initiative de réunir une fois la semaine les élèves du Cours bilingue du High School et de l'Ecole technique, pour des cours de catéchisme. Ils catéchisaient aussi les élèves de l'Institut commercial. Le R.P. Migneault fondait le Camp de Sainte-Anne-des-Chesnes où tous les étés se succèdent des groupes d'enfants.

Cette même année, les plus jeunes filles jouissaient d'une école secondaire catholique sous la direction des Sœurs Grises de la Croix. Si les commencements ont été humbles et pénibles elles jouissent aujourd'hui, grâce à la souscription, d'un édifice moderne bien outillé. 
En 1950, à la demande du Dr Rémi Millette, alors inspecteur des écoles primaires de Sudbury, les Pères de la paroisse formaient l'Association des Parents.

Il ne faut pas oublier les pèlerinages organisés chaque année à la grotte de Lourdes de Sudbury, grotte construite en 1908 par le comte Frédéric Romanet du Caillaud qui devenait la propriété des pères Jésuites le 27 octobre 1951 au coût de $\$ 4,000$.

Mentionnons aussi le clud du Rosaire, le chapelet récité à la radio, tous les soirs, la construction du Chemin de la croix, ainsi que celle du Centre des Jeunes, l'organisation de la section Jeunesses Musicales, la dernière réalisation, la Villa Loyola, endroit d'excursions pour les enfants qui ne peuvent bénéficier d'un séjour au camp, et de la prochaine réalisation, la construction de la Maison des Retraites.

Depuis 1957 la paroisse Sainte-Anne possède un nouveau presbytère et cette année, la vieille église, sous la direction du père Curé actuel, le R.P. Gérard Dallaire, refait sa toilette et modernise ses entrées. Malgré ses 77 ans elle emboîte le pas aussi allègrement que les ramifications auxquelles elle a donné le jour.

\section{Saint-Joseph (Aujourd'hui Christ the King).}

Revenons quelque peu en arrière afin de rappeler l'origine de la paroisse anglaise de Christ the King, première ramification à se détacher de la branche mère.

Les chroniques de l'administration du père Julien Paquin - mars 1914 - juin 1915 - nous relatent clairement les diverses phases de cette première séparation. Parcourons-en rapidement les données. Le père Paquin devait, dès son arrivée, faire face à deux questions délicates: l'agrandissement de l'église et la nécessité de créer une paroisse de langue anglaise. Avec l'esprit de décision et d'exécution du nouveau curé, la première question allait vite se régler; la seconde devait s'orienter doucement vers une solution. Cette lenteur, voulue par le père Paquin était de bonne politique. C'était un projet qui dès l'origine, avait créé une certaine effervescence dans les esprits, de part et d'autres. Il fallait, avant de procéder, laisser au temps le soin de calmer les esprits et les mettre en état de régler la chose sans parti pris.

Dans une réunion, tenue le 13 mai 1914, sous la présidence de $\mathrm{M}^{\mathrm{gr}}$ Scollard, il fut décidé qu'il fallait s'orienter vers l'idée de diviser la paroisse et de créer le plus tôt possible une paroisse de langue anglaise. Le 21 novembre 1914. Monseigneur écrivait au père Paquin qu'il restait favorable à l'idée de la création d'une paroisse de langue anglaise et le 29 mai 1915, il visite et achète un terrain à l'angle des rues Lorne et Pine. Enfin le décret d'érection de la nouvelle paroisse Saint-Joseph ( aujourd'hui Christ the King) fut lu en chaire, le dimanche 16 décembre 1917. Préalablement, soit en $1915, \mathbf{M}^{\mathrm{gr}}$ Scollard avait opter pour le terrain à l'ouest et au nord-ouest du presbytère Sainte-Anne pour y construire l'église et le couvent des Sœurs Saint-Joseph. De plus, à la demande de l'évêque la salle paroissiale Jubilé d'une valeur de $\$ 11,800$ 
et non de $\$ 8,000$ comme on le stipulait, passait à la nouvelle paroisse. C'était une jolie dot de la paroisse mère. Le premier juillet M. l'abbé Crowley, de Copper-Cliff, est nommé curé, et dira la messe pour ses paroissiens dans l'église Sainte-Anne à 9 hres 30, tous les dimanches jusqu'à ce que la Salle Jubilé, transformée en chapelle provisoire, soit prête. Le 23 décembre 1917, $M^{\mathrm{gr}}$ Scollard bénissait la Salle Jubilé devenue temple de Dieu.

Citons quelques extraits tirés de la correspondance d'alors. Le 12 septembre 1917, $\mathrm{M}^{\mathrm{gr}}$ Scollard écrivait: "La division des catholiques de Sudbury en deux paroisses distinctes est basée sur la différence d'idiome ou de la langue plutôt que sur la différence de race. Tout catholique d'origine anglaise, irlandaise, écossaise ou autre nationalité, capable de comprendre et de parler la langue française est libre de s'enrôler au nombre des fidèles de la paroisse Sainte-Anne. Par contre, tout catholique canadien français ou autre nationalité comprenant et parlant la langue anglaise, est libre de se joindre aux fidèles de la paroisse Saint-Joseph. Le salut de vos âmes et surtout le salut des âmes de vos enfants devrait être le motif suprême et prédominant de la décision que vous devez prendre d'appartenir à l'une plutôt qu'à l'autre paroisse. »

Les paroissiens de langue française acceptèrent l'église Sainte-Anne que leur cédait l'évêque tout en acceptant de payer seuls la dette de $\$ 42,000$ mais ils régimbèrent lorsqu'en 1919 des démarches furent faites pour du terrain donné en 1915, en vue d'ériger une salle paroissiale et un orphelinat. Ils se basaient sur le fait que les catholiques de langue anglaise, formant le tiers de la population catholique, auraient un terrain gratuit, une église neuve avec une dette de $\$ 50,000$ pendant que ceux de langue française, formant les deux tiers des catholiques, auraient un terrain gratuit, une vieille église déjà trop petite et une dette de $\$ 42,000$.

Enfin il y eut séparation complète le 10 mars 1920. Jusqu'en 1936 cette nouvelle paroisse porta le nom de paroisse Saint-Joseph pour porter par la suite celui de Christ the King. Elle est aujourd'hui dirigée par son Excellence $\mathrm{M}^{\mathrm{gr}}$ Humphrey, vicaire général du diocèse.

\section{Paroisse Saint-Jean-de-Brébeuf.}

Plusieurs parmi vous, en assistant à la messe d'ouverture de ce Congrès, ont fait connaissance avec la magnifique église Saint-Jean-deBrébeuf. Elle date de 1957 et est placée sous la houlette de son Excellence $\mathrm{M}^{\mathrm{gr}}$ Lorenzo Côté, prélat domestique et vicaire général du Sault Sainte-Marie. Cette paroisse qui comprend environ 1,800 familles, qui jouit de diverses organisations paroissiales telles que: la Ligue du Sacré-Cour, les Congrégations des Dames de Sainte-Anne, des Enfants de Marie, la Congrégation Mariale, les Cercles Lacordaires et Jeanne D'Arc, la Société Saint-Vincent-de-Paul, fut érigée en 1930 pour répondre aux besoins des 30,000 âmes de catholiques du secteur de la ville appelé communément «le moulin à fleur» dans le quartier Fournier. Ses bornes furent déterminées par l'évêque lui-même $\mathbf{M}^{\mathrm{gr}}$ Scollard qui écrit: "Tous les catholiques de langue française qui demeurent des deux côtés des rues Pembroke, Bridge, Leslie et Harold jusqu'à la rue Peter 
inclusivement ainsi que ceux des rues Jogues, Bloor, Kathleen et Melvin seront dorénavant membres de la paroisse Saint-Jean-de-Brébeuf. »

M. l'abbé Joseph-Henri Coallier fut nommé curé fondateur. A peine installé à 216 rue Queen, où il demeura pendant quinze ans, il commença la construction d'un sous-sol. Pendant ce temps ses paroissiens assistaient aux offices divins à l'église Sainte-Anne et au Collège Sacré-Cœur. Pendant 26 ans ces pieux paroissiens à l'esprit de clocher, organisèrent thés, bingos, parties de cartes, au profit de l'église qu'ils désiraient voir

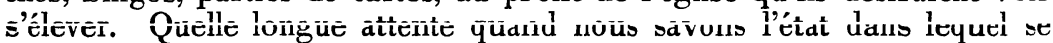
trouvait le sous-sol. Personne dans la région ne peut s'empêcher de penser à la musique bruyante de la pluie tombant du toit dans des chaudières, ainsi qu'aux flaques d'eau dans le chœur.

Enfin en 1956, $\mathbf{M}^{\mathrm{gr}}$ Coallier pouvait annoncer à ses paroissiens que la construction allait commencer à l'été. Atteint d'une grave maladie de cœur, il ne verra que le début des travaux car il s'éteignit le 11 octobre 1956.

C'est à son successeur, $\mathrm{M}^{\mathrm{gr}}$ Lorenzo Côté qu'incombera la lourde tâche de mener à bonne fin la construction de ce temple du Seigneur qui maintenant dresse fièrement vers le ciel son clocher effilé. Nous lui souhaitons de vivre longtemps à l'ombre bienfaisante de son clocher.

\section{Paroisse l'Annonciation.}

On peut dire que la paroisse de l'Annonciation résulte d'un démembrement de la paroisse Saint-Jean-de-Brébeuf. Elle comprend la partie nord de Sudbury et était connue en 1940 sous le nom de North End.

Si à cette date (1940) cette région ne comptait que des fermes et l'école publique $n^{\circ} 4$ fréquentée par des élèves canadiens-français, elle ne fut pas lente à se peupler aussitôt après la cessation de la guerre en 1945. La course à la construction fait bientôt sentir la nécessité pressante de se détacher de la paroisse Saint-Jean-de-Brébeuf.

Par un décret du 28 juin 1950, Rome autorise la fondation de la paroisse de l'Annonciation. La généreuse paroisse mère prête l'un de ses vicaires. Tous les dimanches, le père Brousseau célèbre la messe dans l'école $n^{\circ} 4$ de McKim. Le 10 février 1953 le père Conrad Daigle prend charge de la nouvelle paroisse qui compte déjà 200 familles, chiffre qui se doublera avant la fin de l'année. Trois jours plus tard il aménage la première chapelle au sous-sol d'un logis de la rue Lavoie. Les cents places étant insuffisantes, les paroissiens songent à l'achat d'un terrain pour y construire une église. Le terrain obtenu pour $\$ 100$, les travaux commencent au mois de mai. Grâce à l'aide bénévole des paroissiens qui fournissent près de 4,000 heures de travail, son Excellence $\mathrm{M}^{\mathrm{gr}}$ Dignan pourra bénir officiellement l'église en la belle fête de l'Immaculée Conception, le 8 décembre 1953. La chorale du Collège du Sacré-Cour assume les frais du chant et la cérémonie se termine par la première communion des tout-petits. 
En 1956, le père Jacques Michaud remplace le père Daigle nommé curé à Markstay. Sous l'habile direction de son pasteur actuel la jeune paroisse prend son essor avec confiance et assurance dans l'avenir.

\section{SAINT-EugÈne.}

En 1949 la paroisse Sainte-Anne, comptant alors près de 1,180 familles, réalisait que plusieurs d'entre elles, demeurant à une bonne distance de l'église, fréquentaient les églises anglaises plus rapprochées.

Des patriotes clairvoyants consultèrent le R.P. Alphonse Deguire, alors curé de Sainte-Anne, qui consentit à faire les démarches nécessaires pour obtenir l'érection d'une nouvelle paroisse.

A cet effet cinq paroissiens: MM. Joseph-Armand Lapalme, Alderic Bergeron, Pierre Lebel, Philias Barbeau, et Joseph Samson se cotisèrent pour acheter un terrain de leur choix, situé sur la rue Eyre. Leur prévoyance facilita les transactions quand $\mathrm{M}^{\mathrm{gr}}$ Dignan accorda la permission de construire, le 15 mai 1949. Le R.P. Lorenzo Côté aujourd'hui $\mathrm{M}^{\mathrm{gr}}$ Côté et curé de Saint-Jean-de-Brébeuf fut nommé curé fondateur.

La nouvelle paroisse fut baptisée du nom de Saint-Eugène en l'honneur de Sa Sainteté le Pape Pie XII, Eugène Pacelli, qui célébrait son jubilé d'or sacerdotal, cette année-là.

Le père Côté inaugura le ministère dominical par deux messes célébrées à l'église Sainte-Anne. Le peu d'assistance le força après un mois d'essai d'aller s'installer à la salle de l'Union, rue Régent. Bientôt $\mathrm{M}^{\mathrm{gr}}$ Dignan accorda la permission d'acheter le terrain sur la rue Eyre pour l'érection de la future église. Comme contribution à la nouvelle fondation la paroisse Sainte-Anne verse le prix du terrain, soit \$14,152.

Le 19 septembre, la première messe fut chantée dans la chapelle provisoire au sous-sol du premier presbytère, sur l'autel donné par $\mathrm{M}^{\mathrm{gr}}$ Dignan. En plus, la chapelle possédait 50 sièges, mais le progrès fut rapide, car des bingos et des fêtes champêtres bien organisés, rapportent des recettes dépassant toutes prévisions.

En janvier 1950, Monseigneur accorde la permission de bâtir, bien qu'on entrevoit une dépense de $\$ 50,000$. Les paroissiens, très encouragés par une main-d'œuvre dévouée, semblent vouloir hâter la construction commencée au printemps. Leur rêve se réalise le 6 janvier 1951. En juin, M. l'abbé Marc Boyer, nouvellement ordonné, vient assister le père Côté dans le ministère paroissiale. En octobre 1956, le R.P. Côté, nommé curé à Saint-Jean-de-Brébeuf est remplacé par le R.P. Benoît Fortier.

Ce dernier aura la douleur de voir son église devenir la proie des flammes le samedi de la semaine pascale, 27 avril 1957. Seuls les murs demeurent debouts; il faudra reconstruire. Epreuve terrible. Les messes se disent d'abord au Théâtre Italien mais, par la suite, le service religieux aura lieu au sous-sol de l'école Saint-Albert. Les travaux de construction débutent le jour de la fête de la bonne sainte Anne pour 
se terminer vers le 15 juin 1958, date de la visite officielle de l'église; mais ce n'est qu'à l'automne qu'elle ouvrira de nouveau ses portes au culte, lors de la bénédiction solennelle, par Son Excellence $\mathrm{M}^{\mathrm{gr}}$ Âlexander Carter, qui conféra ensuite la Confirmation aux enfants des écoles de Lockerby, Saint-Alphonse et Saint-Albert.

Cette année, les religieuses des Sœurs Grises de la Croix, qui enseignaient aux écoles Saint-Albert et Saint-Alphonse depuis nombre d'années, deviennent résidentes de la paroisse. Cette jeune paroisse

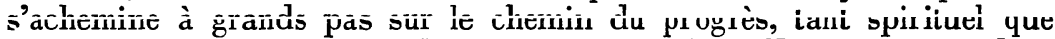
corporel, à la suite de son dévoué pasteur le R.P. Fortier assisté dans son ministère par le R.P. Raymond Prévost, nouveau vicaire.

\section{Lorenzo Deschamps, L.Ph. Président de la Société historique du Nouvel Ontario.}

Faute de temps, nous ne pouvons faire ici l'historique complet de toutes les paroisses catholiques, mais nous tenons au moins à les énumérer. Ce sont:

En 1918, l'église Ukrainienne Sainte-Marie, incendiée et reconstruite en 1928 sur la rue Beech.

En 1936, l'église Saint-Clément, 338 rue Albert, paroisse de 5,986 âmes, sous l'égide de $\mathbf{M}^{\mathrm{gr}}$ Joseph Salini, curé.

En 1938, l'église de la Toussaint, 18 avenue Bellevue, paroisse de 550 familles. Curé fondateur: R.P. Tourangeau; curé actuel: R.P. Fernand Vachon.

En 1938, l'église de Holy Trinity, 4,26 avenue Burton, église polonaise, dirigée par le père Murphy.

En 1939, l'église Notre-Dame-du-Perpétuel-Secours, fondée par le R.P. McCann, 655 rue Lorne, 1,934 âmes, dont le R.P. Brennon est curé.

En 1949, l'église Holy Redeemer, fondée par le R.P. Fitzgerald, 130 Bancroft Drive, dont le R.P. O'Brien est curé.

En 1953, l'église St. Patrick, fondée par le R.P. Thériault, comptant 1,370 paroissiens.

En 1953, l'église Saint-Antoine, 25 rue Fred-Gatchel, fondée par le R.P. Borsa, curé actuel.

En 1955, l'église Saint-Casimir, 210 rue Drinkwater, fondée par le R.P. Jastalski, curé actuel.

En 1956, l'église Saint-Dominique, actuellement en construction, fondée par le R.P. Brosseau; 300 familles ayant comme curé actuel le R.P. Despatie.

$\mathrm{Si}$, aujourd'hui, notre florissante ville de Sudbury, comptant plus de 75,000 âmes, dotée de 16 églises catholiques, d'une Université, de quatre collèges, de 25 écoles promaires, d'une école supérieure et d'une 
école technique, relèvent fièrement la tête et porte haut le flambeau, ne pouvons-nous pas dire qu'elle le doit en majeure partie aux RR. PP. Jésuites qui, nous l'avons vu, à l'instar de nos Saints Martyrs Canadiens, ont su au prix de sacrifices inouis implanter le catholicisme dans cette région.

De nos jours encore, ne continuent-ils pas à assurer son développement en desservant les Missions Indiennes du Sault Sainte-Marie, de Spanish River, de la région de Sudbury et des Iles Manitoulin, en aidant au ministère dominical dans les différentes paroisses où le besoin se fait sentir, en éduquant au Collège du Sacré-Cour, une jeunesse ardente et fière, en éveillant de nombreuses vocations religieuses et sacerdotales et en préparant des professionnels avertis et compétents ? De plus, ne sont-ils pas les promoteurs de nombreux mouvements sociaux et catholiques?

Je crois que je puis me faire le porte-parole de mes compatriotes pour les remercier publiquement, à l'occasion des présentes assises de ce congrès. 